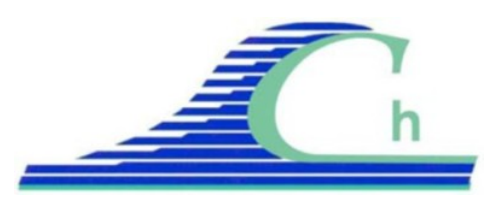

XII ${ }^{\text {ìmes }}$ Journées Nationales Génie Côtier - Génie Civil

Cherbourg, 12-14 juin 2012

DOI:10.5150/jngcgc.2012.041-R C Editions Paralia CFL

disponible en ligne - http://www.paralia.fr - available online

\title{
Modélisation diphasique du transport sédimentaire en régime de Sheet Flow
}

\author{
Thibaud REVIL-BAUDARD ${ }^{1}$, Julien CHAUCHAT ${ }^{1}$
}

\author{
1. Laboratoire LEGI, UMR 5519 1023-1025 rue de la piscine Domaine Universitaire \\ 38400 Saint Martin d'Hères, France. \\ thibaud.revil-baudard@legi.grenoble-inp.fr ; julien.chauchat@hmg.inpg.fr
}

\section{Résumé :}

Le transport sédimentaire en régime de Sheet Flow apparaît lors d'événements extrêmes comme les crues en rivières ou les grosses vagues proches des plages. La compréhension de ce régime est cruciale car il semble être le mode de transport le plus efficace dans la morphodynamique fluviale et côtière. Nous présentons ici un modèle diphasique, eulerien-eulerien, en faisant les hypothèses de vitesses unidirectionnelles, d'uniformité et de régime permanent. Ce modèle est basé sur une rhéologie frictionnelle $(\mu(\mathrm{Iv}))$ pour décrire la relation entre pression et contraintes tangentielles de la phase sédimentaire et sur une loi de décompaction $(\phi(\mathrm{Iv}))$ pour décrire l'évolution de la concentration dans le lit. Ces deux modélisations sont phénomènologiques, basées sur l'analyse dimensionnelle et confirmées par l'expérience. Nous adoptons une approche de type longueur de mélange pour la turbulence fluide avec une loi d'atténuation par rapport à la formulation de Prandtl, le taux d'atténuation dépendant du profil de concentration dans la couche de Sheet Flow. Nous confrontons nos résultats aux expériences de la littérature pour les profils de vitesses et aux résultats d'un autre modèle basé sur la théorie cinétique des écoulements granulaires pour les profils de concentrations. La structure verticale et les paramètres macroscopiques tels que le débit solide, l'épaisseur et la rugosité équivalente de la couche de Sheet Flow sont en bon accord avec les données expérimentales existantes. Une bonne caractérisation de ces paramètres est cruciale pour évaluer la rétroaction du lit mobile sur l'écoulement fluide et pour mieux comprendre le lien entre les mécanismes à l'échelle du sédiment et la morphodynamique à l'échelle de la plage ou de la rivière.

\section{Mots-clés :}

Transport sédimentaire - Sheet Flow - Rugosité - Modèle diphasique - Turbulence Rhéologie frictionnelle - Décompaction - Suspension

\section{Introduction}

Le transport sédimentaire apparaît dans plusieurs phénomènes géophysiques tels que la formation et l'évolution du lit des rivières, les avalanches sous marines ou l'évolution morphologique des plages sous l'effet des vagues. On distingue en général trois modes de transport solide associés à un écoulement fluide sur un lit sédimentaire : le transport 
en suspension, le transport par saltation et le transport par roulement ou charriage sur le fond. La difficulté de l'étude du transport sédimentaire vient de la multitude de paramètres jouant un rôle dans ces processus: granulométrie, rétroaction des grains sur le fluide (viscosité effective, turbulence...), interactions entre les grains (contacts, collisions). Pour caractériser le transport par charriage le nombre de SHIELDS (1936), qui représente la force appliquée par le fluide sur un grain adimensionnée par le poids déjaugé du grain, $\theta=\tau_{\text {bed }} /\left(\Delta \rho g d_{p}\right)$, est classiquement utilisé. Récemment OURIEMI et al. (2009) ont développé un modèle diphasique pour décrire le transport par charriage engendré par un écoulement cisaillé laminaire. L'originalité de ce travail réside dans l'hypothèse que les interactions entre particules sont dues principalement aux forces de contacts entre les grains et sont modélisées par une loi de friction de type Coulomb ou $\mu(\mathrm{Iv})$ (FORTERRE \& POULIQUEN, 2008) telle que celle proposée pour les écoulements de milieux granulaires secs. Nous proposons ici d'étendre ce modèle diphasique au cas où l'écoulement fluide est turbulent et où le lit se décompacte sous l'effet du cisaillement. Dans cette étude nous nous intéressons à un régime particulier du charriage dans lequel on voit apparaître une couche de sédiment transportée d'épaisseur $\delta_{\mathrm{s}}$ est nettement supérieure à la taille des sédiments $d_{\mathrm{p}}$ : le Sheet Flow (SF) ou écoulement en nappe. Ce régime est associé à une très forte contrainte de cisaillement fluide et semble jouer un rôle prédominant dans la morphodynamique fluviale et côtière. Dans le cadre de cette étude, nous modélisons l'écoulement dense en nous limitant à des régimes de Sheet Flow avec peu de suspension $(0.5<\theta<2)$.

\section{Le modèle diphasique}

Le modèle est basé sur la résolution des équations de conservation de la quantité de mouvement et la conservation de la masse pour le fluide et les particules. Afin d'étudier spécifiquement le régime de Sheet Flow, on se place en régime uniforme, en canal de largeur infini, l'écoulement est entraînée par la pente $(\tan \beta)$, on autorise les seules vitesses horizontales, les particules sont toutes des sphères de même diamètre. Dans la suite, les équations pour les vitesses sont projetées dans la direction de l'écoulement $(\mathrm{Ox})$, tandis que les dérivées sont dans la direction verticale $(\mathrm{Oz})$. Dans les équations suivantes, $\mathrm{u}_{\mathrm{f}}$ représente la vitesse fluide et $\mathrm{u}_{\mathrm{p}}$ la vitesse particulaire; $\varepsilon$ et $\phi$ représentent les fractions volumiques respectives du fluide et des particules. La conservation du volume globale impose $\varepsilon+\phi=1$. On peut définir une vitesse de mélange comme la moyenne volumique de la vitesse fluide et de la vitesse particulaire: $U=\varepsilon u^{\mathrm{f}}+\phi u^{\mathrm{p}} . \sigma^{\mathrm{f}}$ et $\sigma^{p}$ représentent respectivement la contrainte tangentielle fluide et particulaire. $\mathrm{P}^{\mathrm{p}}, \mathrm{P}^{\mathrm{f}}, \rho_{\mathrm{f}}$ et $\rho_{p}$ sont les pressions et masses volumiques associées aux deux phases. Les phases interagissent verticalement par la poussée d'Archimède et horizontalement par la traînée et la flottabilité généralisée (JACKSON, 2000).

$0=(1-\phi) \frac{\mathrm{d} \sigma_{x z}^{f}}{\mathrm{~d} z}-C_{D}\left(U-u^{p}\right)+(1-\phi) \rho_{f} g \sin \beta$ 
$0=\frac{\mathrm{d} \sigma_{x z}^{p}}{\mathrm{~d} z}+\phi \frac{\mathrm{d} \sigma_{x z}^{f}}{\mathrm{~d} z}+C_{D}\left(U-u^{p}\right)+\phi \rho_{p} g \sin \beta$

$0=+\frac{\mathrm{d} P^{f}}{\mathrm{~d} z}+\rho_{f} g \cos \beta$

$0=-\frac{\mathrm{d} P^{p}}{\mathrm{~d} z}-\phi\left(\rho_{p}-\rho_{f}\right) g \cos \beta$

Les équations du modèle sont résolues par des schémas aux différences finies. La solution stationnaire du problème est obtenue par intégrations temporelles. Les détails du modèle numérique sont présentés dans REVIL-BAUDARD (2011).

\subsection{La contrainte fluide}

Dans ce problème, la viscosité moléculaire n'est plus le seul mécanisme de diffusion de quantité de mouvement dans le fluide. Les effets de la concentration sédimentaire et de la turbulence sont pris en compte par la somme d'une viscosité effective $\eta_{\mathrm{e}}$ et d'une viscosité turbulente $\eta_{\mathrm{t}}$ :

$\sigma_{x z}^{f}=\left(\eta_{e}+\eta_{t}\right) \frac{\mathrm{d} U}{\mathrm{~d} z}$

La viscosité effective augmente avec la concentration et diverge à la concentration maximum $\left(\phi^{\mathrm{m}} \sim 0,6\right)$ comme le montre la relation (6) (BOYER et al., 2011). Le tenseur de Reynolds des contraintes turbulentes, est modélisé par une viscosité turbulente.

$\eta_{t}=\rho_{f}(1-\phi) l_{m}^{2} \frac{d U}{d z} \quad \frac{\eta_{e}}{\eta_{f}}=1+\frac{5}{2} \phi\left(1-\frac{\phi}{\phi^{m}}\right)^{-1}$

La viscosité turbulente est paramétrée par une longueur de mélange de Prandtl qui représente la taille des structures les plus aptes à transférer la quantité de mouvement. La longueur de mélange est le produit de la constante de Von Karman $(\kappa)$ et de l'intégrale du profil de concentration (LI \& SAWAMOTO, 1995).

$l_{m}=\kappa \int_{0}^{z} \frac{\phi^{m}-\phi}{\phi^{m}} \mathrm{~d} z$

Cette formulation permet d'annihiler la turbulence dans le lit dense et de réduire les effets turbulents dans la couche de Sheet Flow. Dans le cas d'un fluide pur, la longueur de mélange de Prandtl linéaire est retrouvée. La valeur de la longueur de mélange à l'interface représente la hauteur de rugosité $\mathrm{k}_{\mathrm{s}}$ ressentie par la couche limite fluide.

\subsection{La contrainte particulaire}

Suivant le modèle proposé par OURIEMI et al. (2009), nous modélisons la contrainte particulaire avec une rhéologie frictionnelle de type $\mu(\mathrm{Iv})$, FORTERRE \& POULIQUEN (2008). Dans cette approche la contrainte particulaire de cisaillement est proportionnelle à la pression particulaire par le coefficient de friction $\mu(\mathrm{Iv})$ :

$\sigma_{x z}^{p}=\mu\left(I_{v}\right) P^{p}$

Le coefficient de frottement $\mu$ (Iv) évolue d'une valeur statique $\mu_{\mathrm{s}}$ (angle de repos du sédiment), à une valeur dynamique $\mu_{2}$, CASSAR et al. (2005). Cette évolution est 
fonction du nombre visqueux (Iv) qui est le rapport du temps vertical de réarrangement lié à la pression particulaire et à la perméabilité sur le temps horizontal de déformation lié au cisaillement. $I_{0}$ est un paramètre ajustable égal à 1 dans le modèle.

$\mu\left(I_{v}\right)=\mu_{s}+\frac{\mu_{2}-\mu_{s}}{I_{0} / I_{v}+1} I_{v}=\frac{\eta_{f} \frac{d u^{p}}{d z}}{\alpha P^{p}}$ avec $\alpha=\frac{(1-\phi)^{3}}{\phi^{2} 180}$

\subsection{Décompaction et suspension}

Dans le régime de Sheet Flow, deux mécanismes agissent sur la concentration : la dilatance induite par le cisaillement particulaire et la dispersion turbulente. Pour modéliser la variation de concentration liée au cisaillement dans le lit, nous utilisons la formulation suivante obtenue à partir des expériences de BOYER et al. (2011).

$\phi\left(I_{v}\right)=\frac{\phi^{m}}{1+b I_{v}^{a}}$

Dans le reste de l'étude on fixe $b=0,33$ et $a=0,4$. Ces valeurs diffèrent sensiblement de celle de BOYER et al. (2011) : $b=1 ; a=0,5$.

La dispersion turbulente est modélisée dans la couche limite turbulente de l'écoulement fluide par un profil de ROUSE (1938), le poids des sédiments est en équilibre avec la dispersion verticale turbulente.

$w_{s} \phi+\frac{\eta_{t}}{\rho_{f} \sigma_{p}} \frac{\mathrm{d} \phi}{\mathrm{d} z}=0$

où $\omega_{\mathrm{s}}$ est la vitesse de chute et $\sigma_{\mathrm{p}}$ le nombre de Schmidt.

\section{Résultats et discussion}

\subsection{Validation du modèle}

Afin de valider le modèle nous confrontons les profils de vitesses simulés aux profils expérimentaux de COWEN et al. (2010) et de SUMER et al. (1996). Les paramètres physiques et numériques de ces simulations sont récapitulés dans le tableau 1 . Il n'existe pas à notre connaissance de profils de concentration expérimentaux bien résolus dans la littérature, de ce fait les profils de concentration sont confrontés aux résultats du modèle de HSU et al. (2004) basé sur la théorie cinétique des écoulements granulaires.

Les figures 1(a) et 1(b) montrent que dans la zone dense, il existe un bon accord entre les simulations et les profils de vitesse expérimentaux pour différents nombres de Shields. La transition de la zone d'écoulement dense vers la zone plus diluée est bien reproduite par un modèle mixte frictionnel-turbulent. Les profils de concentration prédits par la théorie cinétique semblent en accord qualitatif avec les profils prédits par la loi $\phi(\mathrm{Iv})$, équ. 10. Dans les deux approches une "épaule", ou zone de concentration quasi-constante est prédite. Cela semble confirmer la pertinence d'une approche en $\phi(\mathrm{Iv})$ pour la variation de concentration dans la zone dense et dans la zone de transition vers la suspension. Cependant, le manque de données expérimentales sur le profil de concentration ne nous permet pas de discuter ce résultat plus avant. 


\section{XII èmes Journées Nationales Génie Côtier-Génie Civil}

Cherbourg, 12-14 juin 2012

Tableau1. Paramètres physiques et numériques des simulations

\begin{tabular}{lccccccccc} 
Paramètres & $\theta$ & $\sin \beta$ & $\begin{array}{c}H \\
(\mathrm{~cm})\end{array}$ & $\begin{array}{c}d p \\
(\mathrm{~mm})\end{array}$ & $\rho_{p} / \rho_{f}$ & $\mu_{s}$ & $\mu_{2}$ & Noeuds & $d t(\mathrm{~s})$ \\
\hline COWEN et al. (2010) & 1,25 & 0,0035 & 15,3 & 0,24 & 2,65 & 0,3 & 0,8 & 628 & $10^{-6}$ \\
SUMER et al. (1996) & 1,65 & 0,01 & 9,2 & 2,6 & 1,14 & 0,51 & 1 & 687 & $10^{-5}$ \\
\hline
\end{tabular}

(a)

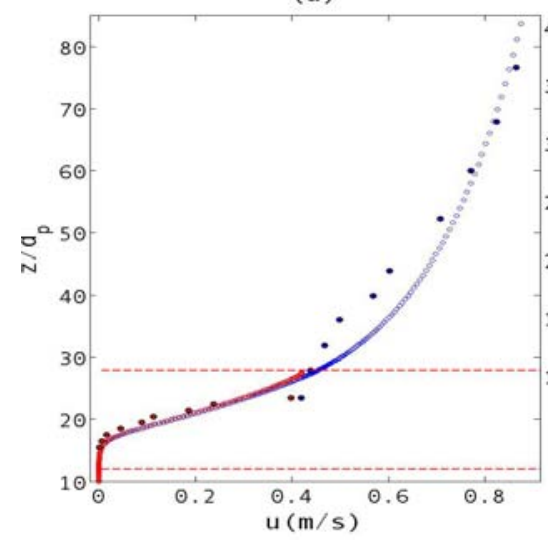

(b)

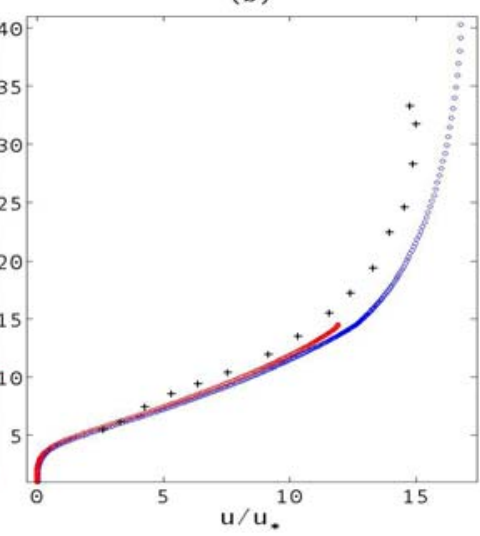

(c)

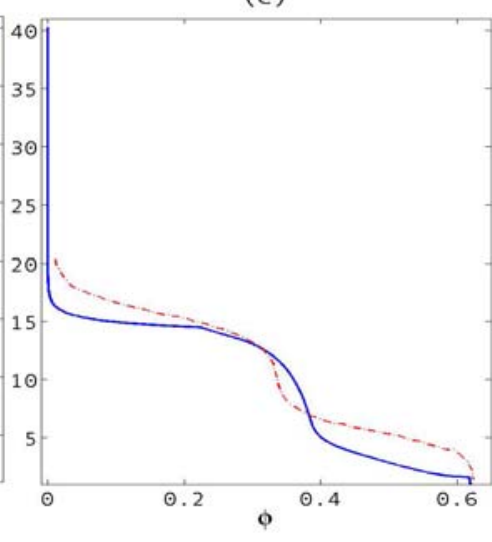

Figure 1. Comparaison entre les profils de vitesses expérimentaux : (a), fluide $(\bullet)$ et solide (•) de COWEN et al. (2010), et (b) de SUMER et al. (1996) (+), avec les profils simulés $\left(o, u_{f} e t o, u_{p}\right)$. Les droites pointillées (- - ) sont les limites de la SF. (c): comparaison entre les profils de concentration simulés par Hsu et al. (2004) (- -) et les simulations du modèle (一).

\subsection{Prédiction des paramètres macroscopiques}

\subsubsection{Débit solide}

La figure 2(a) présente la prédiction du débit solide normalisé, $\left.\psi=\mathrm{Q} /\left(\mathrm{d}\left(\rho_{\mathrm{p}} / \rho_{\mathrm{f}} \mathrm{g} \mathrm{d}_{\mathrm{p}}\right)^{0.5}\right)\right)$ en fonction du nombre de Shields, confrontée aux données expérimentales synthétisées par YALIN (1977). Les résultats des simulations sont en bon accord qualitatif et quantitatif avec les expériences. Les débits obtenus avec le modèle montrent une évolution en loi puissance très proche des formulations proposées dans la littérature $\psi=9(\theta-0,05)^{1.8}$. Le modèle semble prédire un seuil de transport même si l'approche continue n'est pas valide quand l'épaisseur de la couche transportée devient de l'ordre d'un diamètre particulaire. Ces simulations montrent la fiabilité du modèle sur une large gamme d'écoulements et de propriétés sédimentaires.

\subsection{2 Épaisseur de la couche de Sheet Flow}

L'épaisseur de la couche de Sheet Flow est un bon indicateur de la force de l'écoulement et du débit solide associé. Elle dépend de la façon dont le lit va se décompacter et de la 
propension du sédiment ou du mélange à transmettre la quantité de mouvement en profondeur. Comme le montre la figure 2(b), le modèle prédit une évolution linéaire, proche des données expérimentales.

(a)

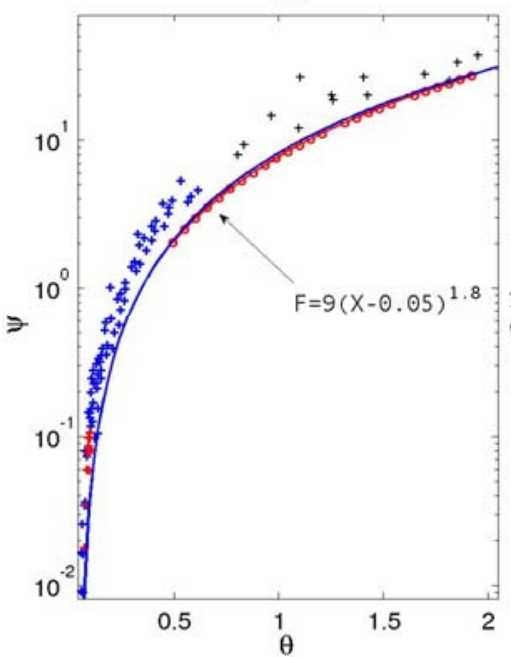

(b)

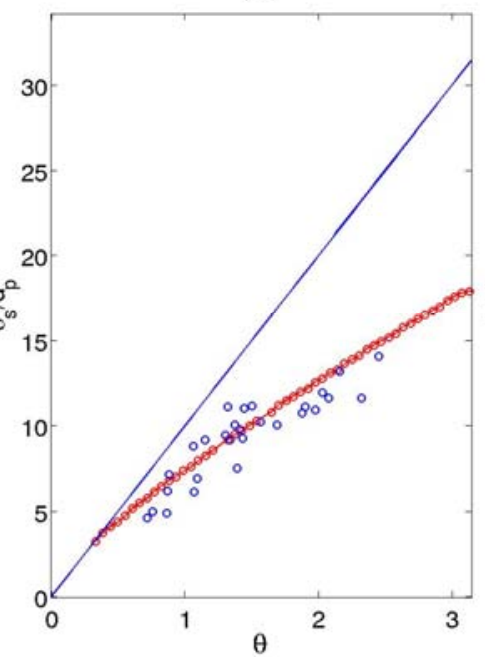

(c)

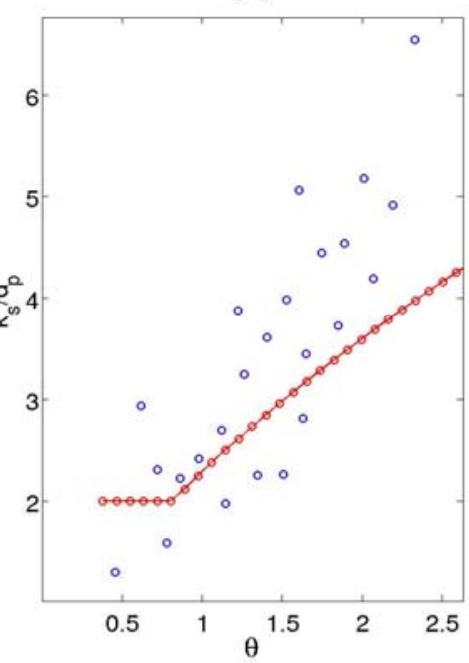

Figure 2. (a) : Comparaison du débit solide adimensionné expérimental (+) et simulé (-o-) en fonction de $\theta$. La courbe(--) représente l'ajustement en loi puissance. (b) : Comparaison entre les épaisseurs adimensionnées expérimentales (o), simulées (-o-) et la relation linéaire de WILSON (1989) (---) en fonction de $\theta$. (c) : Comparaison entre la rugosité adimensionnée par un diamètre particulaire expérimentale (o) et simulés (-o-) en fonction de $\theta$.

\subsubsection{Rugosité}

Dans un écoulement à surface libre, la rugosité contrôle la hauteur de l'écoulement et influe donc sur la contrainte de cisaillement imposée sur le lit et par rétroaction sur le transport lui même. La rugosité est un indicateur des valeurs de perturbations verticales sur l'écoulement horizontal qui initient la formation des tourbillons. La question de la rugosité au dessus d'un fond mobile reste un problème encore assez mal compris. La figure 2(c) montre l'évolution de la rugosité avec le nombre de Shields et on voit une bonne correspondance entre les mesures et les valeurs prédites par le modèle. La rugosité est paramétrée pour être au minimum de 2 diamètres particulaires, ce qui explique la partie constante de la courbe aux faibles nombres de Shields, quand la turbulence est peu efficace dans le lit.

\subsection{Discussion}

La figure 3(a) représente la concentration moyennée sur l'épaisseur de la couche de Sheet Flow en fonction du nombre de Shields. Il est intéressant de voir que la concentration moyenne semble constante sur une large gamme d'écoulement: 


\section{XII ${ }^{\text {èmes }}$ Journées Nationales Génie Côtier - Génie Civil \\ Cherbourg, 12-14 juin 2012}

l'évaluation de l'épaisseur et de la vitesse moyenne serait donc suffisante pour connaître le débit solide. De plus ceci confirme l'idée de BAGNOLD (1956) où il proposait de modéliser la couche de Sheet Flow comme un patin frottant. Ce patin étant mis en mouvement par une forte contrainte fluide appliquée sur la partie supérieur à laquelle résiste la friction à la base du patin. Cette contrainte frictionnelle est paramétrée par le produit du poids du patin et d'un coefficient de friction ad-hoc.

La figure 3(b) montre que la rugosité semble proportionnelle à l'épaisseur de la couche de Sheet Flow (WILSON, 1989). Cela est en accord avec une concentration moyenne constante comme l'avais mesuré (NNADI \& WILSON, 1992). La rugosité caractérise aussi le taux de pénétration de la turbulence dans le lit, qui semble donc proportionnel à l'épaisseur de la Sheet Flow, ce qui n'était pas trivial a priori.

(a)

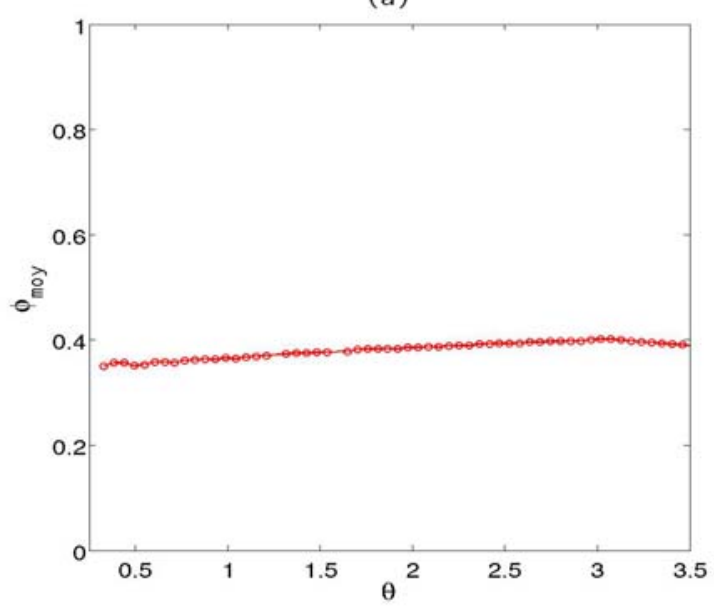

(b)

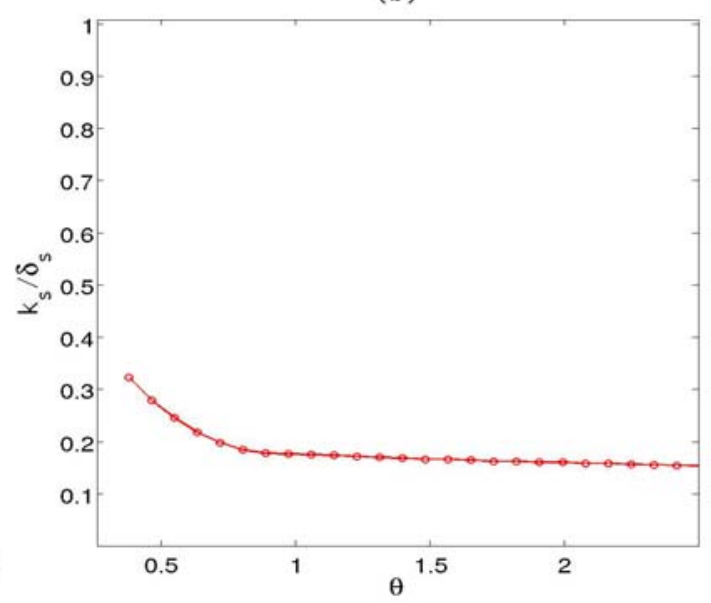

Figure $3:$ (a) : Concentration moyenne dans la couche de Sheet Flow en fonction de nombre de Shields. (b) : Hauteur de rugosité adimensionnée par l'épaisseur de la couche de Sheet Flow.

\section{Conclusion et perspectives}

La confrontation des résultats du modèle proposé à quelques expériences et simulations de la littérature semble valider la pertinence d'une approche mixte frictionnelleturbulente pour l'étude de la structure verticale de la couche de Sheet Flow. Même si certains aspects restent à approfondir, ce type de modèle basé sur une approche phénoménologique frictionnelle semble pertinent pour la prédiction des débits solides, de l'épaisseur de la couche de Sheet Flow et de la rugosité équivalente du fond mobile. Cette étude a montré une certaine robustesse du modèle sur une large gamme d'écoulements et de propriétés sédimentaires.

Des données précises de vitesses et de concentration sont encore manquantes dans ce domaine mais les techniques de mesures modernes (PIV, ADVP, borescope...) devraient permettre dans les prochaines années des avancées significatives. 


\section{Références bibliographiques}

BAGNOLD R.A. (1956). The flow of cohesionless grains in fluids. Phil. Trans. R. Soc. Lond., $249 \mathrm{p}$.

BOYER F., GUAZZELLI E., POULIQUEN O. (2011). Unifying suspension and granular rheology. Phys. Rev. Lett., Vol. 107(18), 188301. doi:10.1103/PhysRevLett.107.188301 CASSAR C., NICOLAS M., POULIQUEN O. (2005). Submarine granular flows down inclined planes, Physics of Fluids, 17(10), 103301. doi:10.1063/1.2069864

COWEN E.A., DUDLEY R.D., LIAO Q., VARIANO E.A., LIU P.L.-F. (2010). An insitu borescopic quantitative imaging profiler for the measurement of high concentration sediment velocity. Experiments in Fluid, Vol. 49(1), pp 77-88. doi:10.1007/s00348-009-0801-8

FORTERRE Y., POULIQUEN O. (2008). Flows of dense granular media. Annual Review of Fluid Mechanics, Vol. 40, pp 1-24, doi:10.1146/annurev.fluid.40.111406.102142

HSU T., JENKINS J.T., LIU L.F. (2004). On two-phase sediment transport : sheetflow of massive particles. Proc. R. Soc. Lond. A, Vol. 460, pp 2223-2250. doi:10.1098/rspa.2003.1273

JACKSON R. (2000). The Dynamics of Fluidized Particles. Cambridge University Press.

LI L., SAWAMOTO M. (1995). Multi-phase model on sediment transport in sheet-flow regime under oscillatory flow. Coastal engineering Japan, Vol. 38, pp 157-178.

NNADI F.N., WILSON K.C. (1992). Motion of contact-load particles at high shear stress. Journal of Hydraulic Engineering, Vol. 118(12), pp 1670-1684, doi:10.1061/(ASCE)0733-9429(1992)118:12(1670)

OURIEMI M., AUSSILlOUS P., GUAZZELLI E. (2009). Sediment dynamics. Part I : Bed-load transport by shearing flows. Journal of Fluid Mechanics, Vol. 636, pp 295-319. doi:10.1017/S0022112009007915

REVIL-BAUDARD T. (2011). Modélisation diphasique du transport sédimentaire en régime de Sheet Flow. Rapport de stage M2R au LEGI, 30 p.

ROUSE H. (1938). Experiments on the mechanics on sediment suspension, Trans. Am. Soc. Civ. Eng., 102, pp 463-505.

SHIELDS A. (1936). Application of similarity principles and turbulence research to bed-load movement. Mitteilunger der Preussischen Versuchsanstalt für Wasserbau und Schiffbau, Vol. 26, pp 5-24.

SUMER B.M., KOZAKIEWICZ A., FREDSOE J., DEIGAARD R. (1996). Velocity and concentration profiles in sheetflow layer of movable bed. Journal of Hydraulic Engineering, Vol. 122:10(549) (10 pages). doi:10.1061/(ASCE)0733-9429(1996)122:10(549)

WILSON K.C. (1989). Mobile-bed friction at high shear stress. Journal of Hydraulic Engineering, Vol. 115 (6), pp 825-830. doi:10.1061/(ASCE)0733-9429(1989)115:6(825) YALIN M.S. (1977). Mechanics of sediment transport. 2nd edition, Pergamon Press, Ontario. 